\title{
PUBLICACIÓN DE MONOGRAFÍAS EN ACCESO ABIERTO POR EDITORIALES UNIVERSITARIAS ESPAÑOLAS
}

\author{
Open access monographs published by university \\ presses in Spain
}

Ernest Abadal, Candela Ollé y Sílvia Redondo

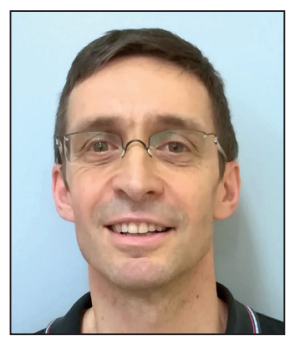

Ernest Abadal es catedrático de la Facultat de Biblioteconomia i Documentació de la Universitat de Barcelona habiendo ejercido como decano de esa facultad en el período 2011-2018. Dirige un proyecto de investigación sobre acceso abierto a la ciencia en España, es coordinador del grupo de investigación consolidado Cultura y Contenidos Digitales y de la red temática Maredata (red sobre datos de investigación en abierto). Es miembro del consejo asesor de diversas revistas científicas. http://www.accesoabierto.net

http://orcid.org/0000-0002-9151-6437

Universitat de Barcelona Facultat de Biblioteconomia i Documentació Melcior de Palau, 140. 08014 Barcelona, España abadal@ub.edu

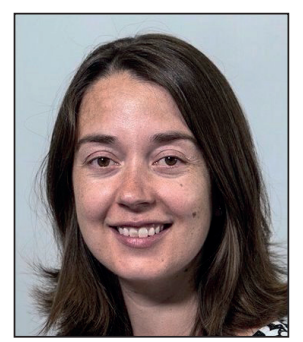

Candela Ollé es doctora en Documentación por la Universitat de Barcelona y licenciada en Periodismo por la Universitat Autònoma de Barcelona. Profesora de los Estudios de Comunicación y Ciencias de la Información de la Universitat Oberta de Catalunya (UOC), gestora de la red temática Maredata y codirectora de la revista BiD: textos universitaris de biblioteconomia i documentació. Sus líneas de investigación se centran en el comportamiento informacional de los usuarios, uso de revistas y libros electrónicos, movimiento de acceso abierto en las universidades españolas, y productividad y género.

http://orcid.org/0000-0002-8302-4790

Universitat Oberta de Catalunya Estudis de Comunicació i Informació Av. Tibidabo, 39-43. 08035 Barcelona, España collec@uoc.edu

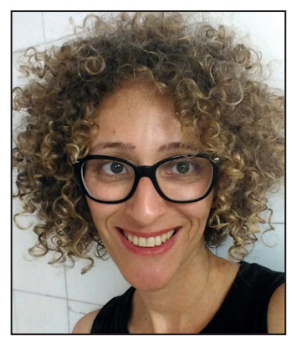

Sílvia Redondo es licenciada en Historia y en Documentación. Ha trabajado como profesora asociada y documentalista en la Universitat de Barcelona y también en la Xarxa de Biblioteques Públiques de la Diputació de Barcelona como directora de biblioteca. Colabora con el grupo de investigación Acceso Abierto y es responsable del Ámbito de Información e Investigación en la Fundació Escoles Garbí. Sus líneas de investigación son el acceso abierto y la difusión de la investigación científica. http://orcid.org/0000-0002-5151-0903

Fundació Escoles Garbí Rambla Catalunya, 17, $3^{r} 2^{\mathrm{a}}$. 08007 Barcelona, España sredondo@escolesgarbi.cat

\section{Resumen}

Se analiza la situación del modelo de acceso abierto para la publicación de monografías de las editoriales académicas españolas integrantes de la Unión de Editoriales Universitarias Españolas (UNE). Se presentan los datos de productividad del período 2015-2017, así como el posicionamiento y argumentos de la dirección de las editoriales, políticas, y modalidades de financiamiento. Para la recogida de datos se envió un cuestionario a los editores asociados a UNE (respondido por el 58\%), complementado con entrevistas en profundidad a siete de ellos. Los resultados muestran que el $75 \%$ de los editores publica títulos en acceso abierto. La mayoría opina que el acceso abierto incrementa la difusión de las monografías (tanto consultas como descargas) y que no existen diferencias en la calidad de los contenidos. Los editores no ven claro que sea compatible el acceso abierto con la explotación comercial de ejemplares impresos, y consideran que las vías de financiación más efectivas son la institucional (a cargo de la universidad), seguida por la propia editorial y el pago por parte del autor. 


\title{
Palabras clave
}

Editoriales universitarias; Acceso abierto; Monografías; Encuestas; Cuestionarios; Entrevistas; Financiación; Políticas; Unión de Editoriales Universitarias Españolas; UNE.

\begin{abstract}
The open access model for publishing monographs by the members of the Spanish Universities Publishers Association (UNE) is analyzed. The study focused on production data from 2015-2017, as well as the positioning and arguments of the editors in relation to the open access movement, publication, related policies, and financing modalities. Data was collected by a questionnaire (with a response rate of $58 \%$ of the publishers associated with UNE) and includes in-depth interviews with seven of them. The results show that $75 \%$ of UNE members publish titles in open access, most consider open access a good way to increase the dissemination of monographs, and that there are no differences in content quality with non-open access works. Publishers do not see that publishing in open access is compatible with the commercial exploitation of printed copies. Publishers consider the most effective financing channels to be institutional payment (i.e., the university), followed by publisher's self-financing, and payment by the author.
\end{abstract}

\section{Keywords}

University publishers; University presses; Open access; Monographs; Surveys; Questionnaires; Interviews; Funding; Policies; Spanish Universities Publishers Association; UNE.

Abadal, Ernest; Ollé, Candela; Redondo, Sílvia (2018). "Publicación de monografías en acceso abierto por editoriales universitarias españolas". El profesional de la información, v. 27, n. 2, pp. 300-311.

https://doi.org/10.3145/epi.2018.mar.08

\section{Introducción}

El incremento de la difusión de los contenidos académicos se ha visto propulsado en los últimos años por los procesos de digitalización y también por la difusión en acceso abierto. Esto se ha hecho patente especialmente en los artículos de revista, una buena parte de los cuales ya son accesibles de manera libre y gratuita a todos los usuarios en repositorios o portales de revistas en unos porcentajes que oscilan entre el 20 y el 50\%, según diversos estudios (Björk et al., 2010; Archambault et al., 2013; Chen, 2014).

A pesar de la importancia que tienen las monografías en la edición académica el grado de difusión de sus contenidos es menor que el de los artículos de revista. ¿Por qué? ¿Cuáles son los motivos que explican esta diferencia? El menor grado de digitalización de las monografías seguramente sea una de las causas. Pero especialmente esto se debe, en nuestra opinión, a que prácticamente no se ha implementado el modelo de acceso abierto en los libros. Tres proyectos europeos sobre el futuro de la publicación de monografías han generado diversos estudios sobre esta cuestión:

- el informe final del proyecto Oapen (Open access publishing in European networks) que analizó las vías de sostenibilidad para monografías académicas en ciencias humanas y sociales en Europa (Collins; Milloy, 2016), y que realizó también un análisis específico en Países Bajos (Ferwerda; Snijder; Adema, 2013);

- el proyecto Knowledge unlachted, que se basa en el establecimiento de un consorcio mundial para el sostenimiento de la publicación de monografías (Montgomery, 2015); - el proyecto The academic book of the future project, financiado por el Arts and Humanities Research Council $(A H R C)$ y la British Library $(B L)$ que generó dos informes finales complementarios (Deegan, 2017; Jubb, 2017) que analizan la situación y las perspectivas de futuro del libro académico desde el punto de vista de los diversos agentes (editores, biblioteca universitaria, libreros, etc.) y también de los procesos (publicación, difusión, contratos, ventas, etc.) incluyendo un capítulo dedicado al acceso abierto.

De manera complementaria podemos citar también una interesante investigación sobre modelos de financiación (Ferwerda, 2014), un estudio sobre el futuro de las monografías en la era digital (Elliott, 2015) que destaca el papel del acceso abierto, y un informe sobre las políticas de las monografías en acceso abierto, las fuentes de financiación y los modelos de publicación en ocho países europeos a partir de 73 conversaciones en profundidad, con editores, financiadores y bibliotecas (Ferwerda; Pinter; Stern, 2017).

Desde nuestro punto de vista, las editoriales universitarias - un tipo específico que concentra la práctica totalidad de su producción en publicaciones académicas, principalmente monografías pero también revistas científicas - reúnen las condiciones para adoptar el modelo de acceso abierto en monografías, dado que tienen una dependencia institucional de las universidades (o de centros públicos de investigación, como sería el caso del $(S I C)$, muchas de las cuales disponen de políticas de acceso abierto.

La situación de la edición universitaria en España ha sido estudiada con anterioridad de manera general (Polo-Pujadas, 2007; Abadal; Ollé, 2012) o centrándose en el libro electrónico (Cordón-García, 2014; Cordón-García et al., 2013). Las editoriales universitarias constituyen el primer grupo de edición académica de España, por delante de otros como Hachette o Planeta, ya que en 2015 publicaron el 30\% de los libros académicos (el 33,6\% del fondo vivo). Los datos estadísticos actualizados sobre la publicación anual se pueden encontrar en Las editoriales universitarias en cifras (UNE, 
2016), basado en encuestas a los editores y con apartados referentes a la edición, venta, perfil de los lectores, derechos de autor, intercambios con otras instituciones, personal, colaboradores y publicidad. En la última edición, además, se incluyó alguna pregunta sobre acceso abierto. Las características generales más destacadas son el predominio ciencias sociales y humanidades en los títulos publicados (75\%) y un notable incremento de las ediciones digitales.

En lo que se refiere a estudios específicos sobre editoriales universitarias y acceso abierto, podemos destacar

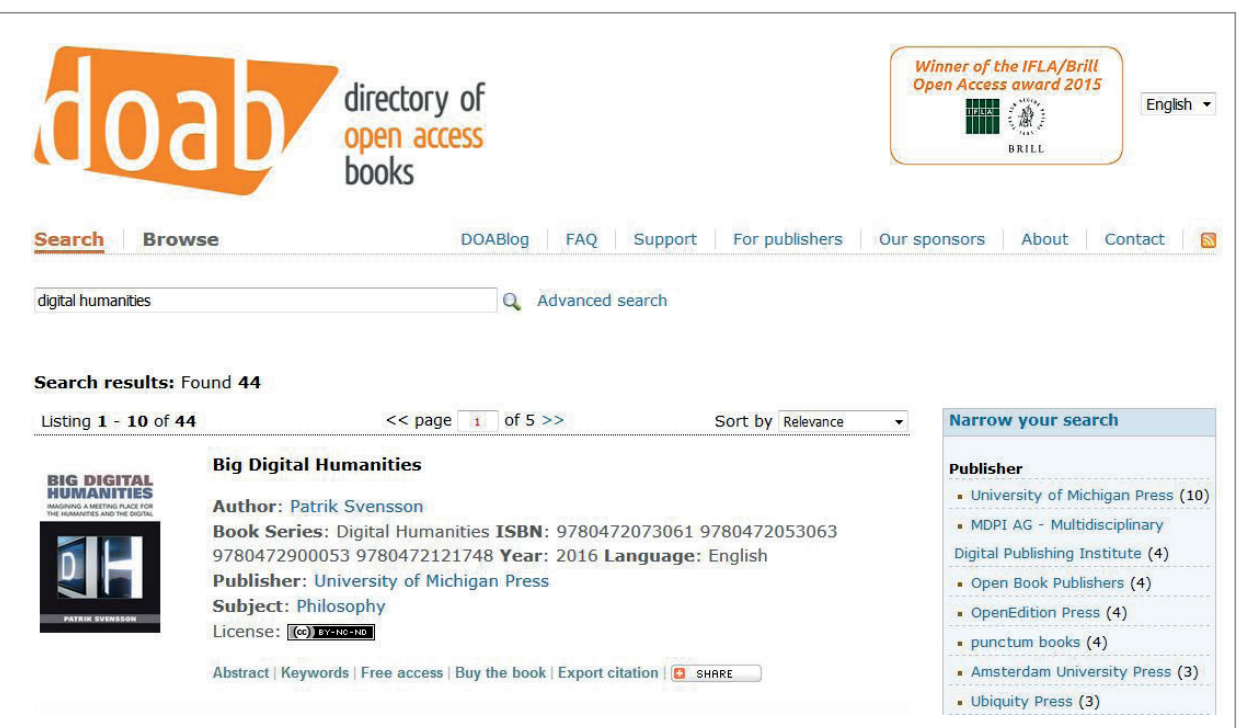

Figura 1. Directory of open access books http://www.doabooks.org el texto pionero de Greco (2008) en el que se preguntaba sobre la conveniencia de que las editoriales universitarias adopten este modelo para toda su producción. También se dispone de un par de estudios de campo que se centran, curiosamente ambos, en el mismo país: Shearer (2010) llevó a cabo un análisis detallado de los nuevos modelos editoriales de las universidades de Canadá e incluyó un apartado dedicado a la publicación de monografías donde indica además algún ejemplo de buenas prácticas. Taylor et al. (2013) realizaron un estudio similar al anterior, aunque centrado en revistas, que partió de una encuesta a gestores de publicaciones universitarias de Canadá y a directores de bibliotecas -aunque obtuvo un número de respuestas de editores más bien bajo (6 de 19)-.

Finalmente, nos tenemos que referir a las plataformas de difusión de las monografías entre las cuales destaca Oapen, antes citado, ya que contiene unos 500 libros de humanidades y otros tantos en ciencias sociales editados por prestigiosas universidades (como Amsterdam University Press, Leiden University Press, Göttingen University Press, Manchester University Press, Presses Universitaires de Lyon, y Firenze University Press). Como en el caso de las revistas, mantienen un modelo de calidad muy claro: sólo se publican aquellos textos que han superado un proceso de revisión externa por pares (peer-review). El modelo de negocio que permite subsistir a este tipo de edición es la combinación del acceso abierto (en formato pdf, normalmente) con la venta de los libros en otros formatos (impreso, ebook, etc.). En algún caso, además, también se solicita al autor el pago de los costes de edición.

También puede consultarse el Directory of open access books que contiene información sobre unos 7.500 libros y capítulos, publicados por 225 editores, unas cifras aún alejadas de las 10.000 revistas y unos $2,6 \mathrm{mi}$ llones de artículos que constan en el directorio homólogo (Directory of open access journals) dedicado exclusivamente a revistas. Como se puede constatar, las modestas cifras de libros disponibles en acceso abierto no se pueden comparar con los datos cuantitativos de las revistas.

http://www.doabooks.org

La editorial Australian National University Press (ANU Press), creada en 2003, ha sido una de las pioneras en la edición en acceso abierto. Actualmente dispone de un catálogo de unos 600 títulos, con la posibilidad de descarga gratuita en e-book y pago por la versión impresa.

\section{Buy print $(\$ 50.00)$}

Figura 2. Consulta al catálogo de ANU Press

https://press.anu.edu.au 
En España no existen proyectos similares, y además los editores hispanos tienen escaso interés en participar en los proyectos internacionales antes señalados. La excepción sería Edit.um (Ediciones Universidad de Murcia), que ha puesto en marcha el portal Libros.um en el que se puede descargar el pdf de más de 150 títulos de todas las disciplinas. También es verdad que en algunos casos se utilizan los repositorios institucionales para la difusión de las monografías, aunque raramente se dispone de las colecciones destacadas y, por tanto, la visibilidad de los títulos queda muy disminuida, en especial si la comparamos con los portales antes descritos.

El objetivo general de nuestro estudio es analizar la situación actual del modelo de acceso abierto para la publicación de monografías por parte de editoriales universitarias españolas. Los objetivos específicos han sido los siguientes:

a) Conocer las características de las editoriales que publican en acceso abierto.

b) Conocer cuáles son sus planes de futuro a corto y medio plazo respecto de este modelo de edición.

c) Conocer la valoración sobre las ventajas e inconvenientes de la publicación de monografías en acceso abierto por parte de los editores.

d) Conocer su valoración sobre las posibles vías de financiación.

e) Determinar si existe relación entre la publicación en abierto y las políticas de la universidad.

\section{Material y métodos}

La metodología utilizada ha sido, en una primera fase, una encuesta preparada con una mayoría de preguntas cerradas y algunos campos abiertos (ver anexo). A continuación, los encuestados que mostraron interés en seguir colaborando con el estudio fueron entrevistados de manera presencial, con un cuestionario semi estructurado, en la segunda fase.

La encuesta se envió con la colaboración de la Unión de Editoriales Universitarias Españolas (UNE) el 8 de noviembre de 2016. Se realizaron tres recordatorios, el primero (29 de noviembre) a cargo de la secretaría de la UNE y los otros dos (10 de enero y 14 de febrero de 2017) por los autores del artículo. Obtuvimos 32 respuestas de un total de 55 editoriales, una tasa de respuesta del 58\%.

La encuesta consta de cuatro apartados:

- datos descriptivos;

- valoración del acceso abierto en la publicación de monografías;

- publicaciones en acceso abierto;

- modelos y políticas de publicación en acceso abierto;

y un campo abierto para comentarios finales.

Los indicadores considerados han sido:

- número de títulos publicados;

- formato de publicación (papel, digital, ambos);

- publicación en acceso abierto o no;

- tema de los títulos;

- datos sobre el incremento o no de las consultas;

- mantenimiento de la calidad de los contenidos;
- explotación comercial de las obras;

- volumen de descargas;

- viabilidad económica.

Las entrevistas se realizaron a lo largo de enero de 2017 a siete responsables de editoriales universitarias ${ }^{1}$ para profundizar en el sistema de publicación de cada una, conocer su planificación de publicación de monografías en acceso abierto a corto y medio plazo, así como detectar las dudas y su posicionamiento en relación con el acceso abierto, entre otros aspectos. Todos ellos aceptaron constar en el informe final y han revisado y aceptado la redacción final de este manuscrito.

\section{Resultados}

A continuación presentamos el análisis de los datos extraídos del cuestionario, complementados en algunos casos con las declaraciones procedentes de las entrevistas individuales.

\subsection{Datos generales}

En lo que se refiere a la dimensión de las editoriales, una cuarta parte de ellas se pueden considerar grandes ( 8 de las 32 editoriales universitarias que han contestado el cuestionario han publicado más de 100 títulos tanto en 2015 como en 2016). En la tabla 1 podemos ver que la concentración se produce en la franja de editoriales que han publicado menos de 50 títulos por año, con un 53\% (17) en 2015 y un $50 \%$ (16) en 2016. En global se aprecia una estabilidad del volumen de títulos y se apunta una ligera tendencia al alza del grupo de 50 a 100 títulos que sería conveniente contrastar con los datos al final de 2017. Podemos apuntar que en relación con el tamaño de las editoriales, considerando su volumen de producción, la mitad de ellas son más bien pequeñas (menos de 50 títulos) repartiéndose el resto a partes iguales entre grandes y medianas editoriales.

En lo que respecta al formato de publicación (figura 3) se preguntó el porcentaje de los títulos publicados en papel, en digital o ambos (papel y también digital) y se les ofrecía escoger las opciones siguientes: de 0-25\%; 26-50\%; 51-75\% y $76-100 \%$, motivo por el cual el sumatorio no es 100 . Se constata que dos tercios de los editores publican la mayor parte de sus títulos en papel y una cuarta parte ( $25 \%$ de las editoriales) publica la mayoría de sus títulos en formato digital, aunque también destaca el volumen de publicación en ambos formatos. Las variaciones en los dos años analizados son mínimas. El ligero descenso de la publicación en papel se corresponde con el aumento de las versiones digitales.

Los responsables de las editoriales entrevistadas - como es el caso de la Universitat de València (UV) o de la Universidad

Tabla 1. Panorámica de la publicación de títulos en los años 2015-2016

\begin{tabular}{|l|c|c|}
\hline \multirow{2}{*}{$\begin{array}{c}\text { Número de títulos } \\
\text { publicados }\end{array}$} & \multicolumn{2}{c|}{ Porcentaje de editoriales } \\
\cline { 2 - 3 } & $\mathbf{2 0 1 5}$ & $\mathbf{2 0 1 6}$ \\
\hline$>100$ & 25 & 25 \\
\hline de 50 a 100 & 22 & 25 \\
\hline$<50$ & 53 & 50 \\
\hline
\end{tabular}




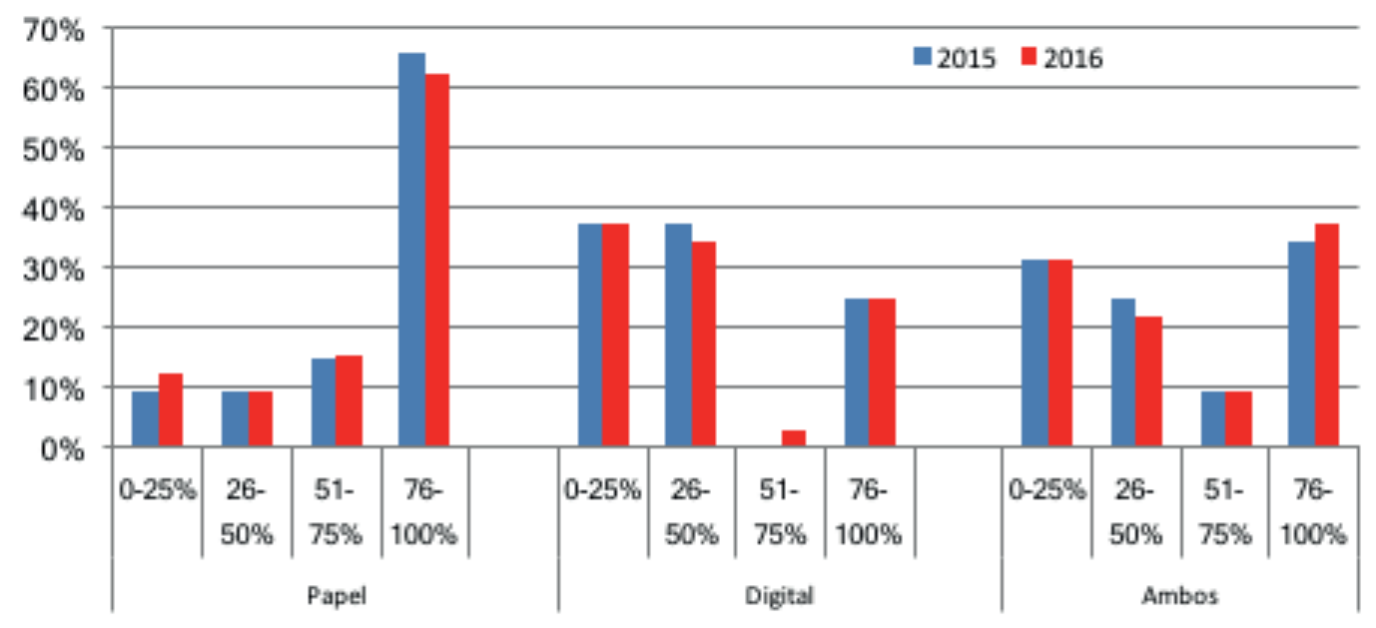

Figura 3. Títulos publicados según formato

Complutense de Madrid (UCM) - sostienen que los autores aún quieren ver sus obras en papel.

Aunque no se pueden establecer paralelismos directos con nuestra tabla, podemos tomar también como referencia la encuesta de UNE (2016) antes señalada, donde se indica que el $33 \%$ de la producción editorial universitaria se encuentra accesible en formato digital (teniendo en cuenta que el $88,1 \%$ que se edita en digital se publica también en papel).

El $59 \%$ de las editoriales encuestadas publican algún título en acceso abierto y, de cara a 2017 , un $28 \%$ de las que no lo hacían, estaban valorándolo

\subsection{Publicación en acceso abierto}

En este apartado vamos a hacer referencia a las editoriales que han publicado en acceso abierto y a la distribución temática de los títulos.

En primer lugar es importante destacar que el 59\% de las editoriales encuestadas publican algún título en acceso abierto y que, de cara a 2017 , un $28 \%$ de las que no lo hacían estaban valorando la posibilidad de difundir libros bajo este modelo. Se trata de una proporción especialmente relevante aunque sea un poco inferior al $67 \%$ que consta en los datos estadísticos de UNE (2016). Hay que tener en cuenta que la pregunta que se formulaba allí incluía no sólo los libros (como es nuestro caso) sino también las revistas.

Publicacions de la Universitat Rovira i Virgili (URV) es un caso destacable dado que publican todos sus títulos en acceso abierto desde el primer momento. Otro grupo de editoriales (UPV, UPC, etc.) publica en abierto una buena parte de sus títulos y deja el resto con un embargo de dos o tres años. También está el caso de Editorial UOC que probó este modelo y no les funcionó, regresando entonces al sistema comercial tradicional.
Las dificultades para implementar el modelo de acceso abierto de las monografías han sido también comentadas por el editor de la UV:

"La evolución de las monografías en acceso abierto no será como el de las revistas; será más lento porqué el coste de publicación es más alto y, además, hay una tradición: la gente, sobre todo los catedráticos y titulares de humanidades, quiere ver su libro en papel".

Las editoriales que ofrecen los libros en acceso abierto lo justifican por el incremento de la difusión, porque así lo indica su política editorial o porque es una forma de prolongar la vida del libro una vez finalizado su recorrido comercial.

En lo que respecta al número de títulos publicados en acceso abierto y a su distribución temática, tan sólo 4 editoriales compartieron los detalles. A partir de esta información, las disciplinas que encabezaban la publicación en acceso abierto en 2016 eran ciencias sociales $(29,6 \%)$ y humanidades $(27,8 \%)$, seguidas de ingeniería $(14,8 \%)$, ciencias experimentales $(16,7 \%)$ y matemáticas (11\%). No se indican títulos en ciencias de la salud y, por otra parte, los porcentajes son casi idénticos para 2015 y 2016 (figura 4).

Se trata de una distribución temática similar a los porcentajes de la publicación global de títulos de las editoriales universitarias (UNE, 2016) excepto para el caso de las humanidades que suponen el $46 \%$ de los títulos publicados, pero sólo el $25 \%$ de los que se pueden encontrar en acceso abierto. Esta diferencia se explica por tratarse de un campo con unos hábitos donde predomina el uso del papel y con mayores reticencias por parte de los autores (v. 3.3).

Además de la opción de publicar en acceso abierto, existe la posibilidad de "liberar" los títulos que han terminado su etapa de explotación comercial indicada en el contrato de edición. En este sentido, un $38 \%$ de las editoriales afirman ponerlos en acceso abierto, un $28 \%$ no lo hacen y un $34 \%$ lo está valorando. En las explicaciones indicadas en la pregunta abierta vinculada, las editoriales que no optan por el acceso abierto tras el período de explotación señalan que el principal obstáculo es la inexistencia en el contrato de una 


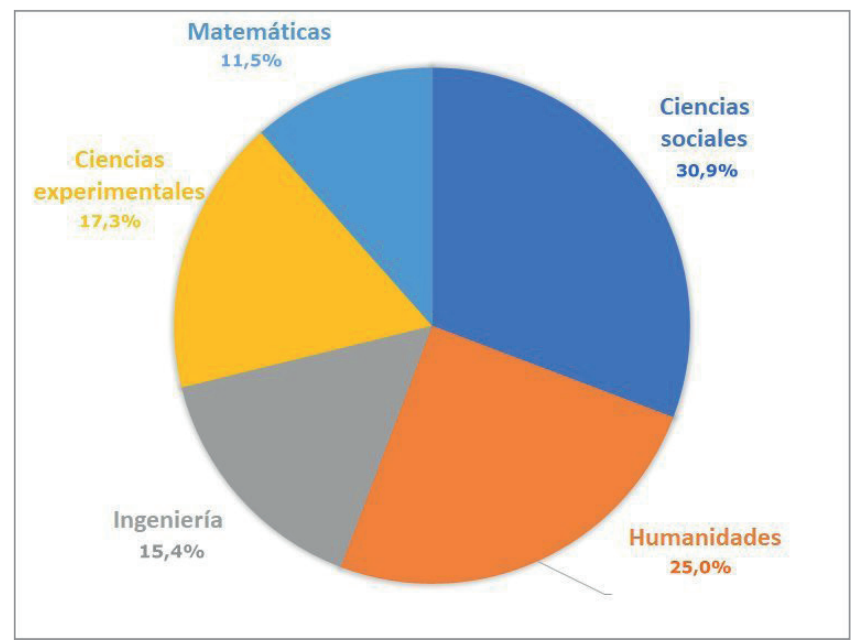

Figura 4. Disciplinas de los libros publicados en acceso abierto en 2016

cláusula que así lo especifique. Tampoco se plantean modificar el contrato para incluir esta posibilidad porque aseguran que no es la política de la editorial y, además, el autor normalmente prefiere renovar la cesión de los derechos de explotación para seguir vendiendo su libro. En el caso de la UV - que no publican en acceso abierto- cuando finaliza el contrato y si el autor lo pide se le entrega el formato electrónico para que lo ponga en el repositorio institucional. Este sistema lo utilizan especialmente en los libros colectivos, con ventas muy escasas dado que los lectores se procuran por otros medios la separata que les interesa.

A partir de las entrevistas se observa una mayor predisposición a publicar monografías en acceso abierto en las editoriales de las universidades politécnicas (UPV y UPC). En ambos casos afirman que sus modelos de publicación son totalmente sostenibles.

En algunas editoriales se tiene un concepto un tanto laxo del acceso abierto ya que éste no se ciñe a la doble exigencia de gratuidad y cesión de derechos, sino que se centra exclusivamente en la primera de ellas. Este es el punto de vista defendido por el representante del CSIC:

"Hemos encontrado una vía que se centra en distinguir gratuito de open access. Publicar en acceso abierto conlleva aplicar una licencia Creative Commons y por tanto, como editorial, la renuncia a una serie de derechos. La gratuidad sólo se refiere a cuestiones económicas. Nuestra intención es facilitar el acceso al conocimiento, y la vía que utilizamos es distribuir gratuitamente en nuestra web una selección de títulos, pero sin cesión de derechos. Es un archivo pdf que lleva la mención '(c) CSIC. (c) del autor o autores / Todos los derechos reservados. Copia gratuita'”.

\subsection{Valoración sobre el acceso abierto}

Después de conocer la realidad de la publicación en abierto, interesó saber las opiniones de los directores de las editoriales respecto de las supuestas ventajas que se atribuyen a la publicación en acceso abierto, en especial al incremento de consultas, al mantenimiento de la calidad de los contenidos y a la posibilidad de compatibilizarlo con una explotación comercial de la versión impresa. A las preguntas se tenía que responder con una valoración (si están totalmente de acuerdo, de acuerdo, ni en acuerdo ni en desacuerdo, en desacuerdo, totalmente en desacuerdo).

Las editoriales que ofrecen los libros en acceso abierto lo justifican por el incremento de la difusión, por su política editorial o porque es una forma de prolongar la vida del libro una vez finalizado su recorrido comercial

Un 75\% de los editores considera que el acceso abierto es una buena actuación para incrementar la difusión de las monografías (consultas y descargas). Existe una correlación muy alta entre las editoriales que publican en acceso abierto o lo están valorando y la consideración positiva a esta pregunta.

La mayoría de los comentarios positivos aluden al incremento de visibilidad tanto del autor como de la institución a la que pertenece la editorial, a la rapidez en el acceso, a la ausencia de coste, a la posibilidad de su reutilización por parte de los ciudadanos, y algunos también aportan cifras concretas de incremento de consultas en sus estadísticas. Así, por ejemplo, la UPC considera que:

"las consultas y/o descargas aumentan después de ponerlo en acceso abierto",

y la URV afirma que:

"publicar en abierto permite aumentar la visibilidad y las citas".

En el extremo opuesto, los menos partidarios expresan que no tienen evidencias y que depende de los temas.

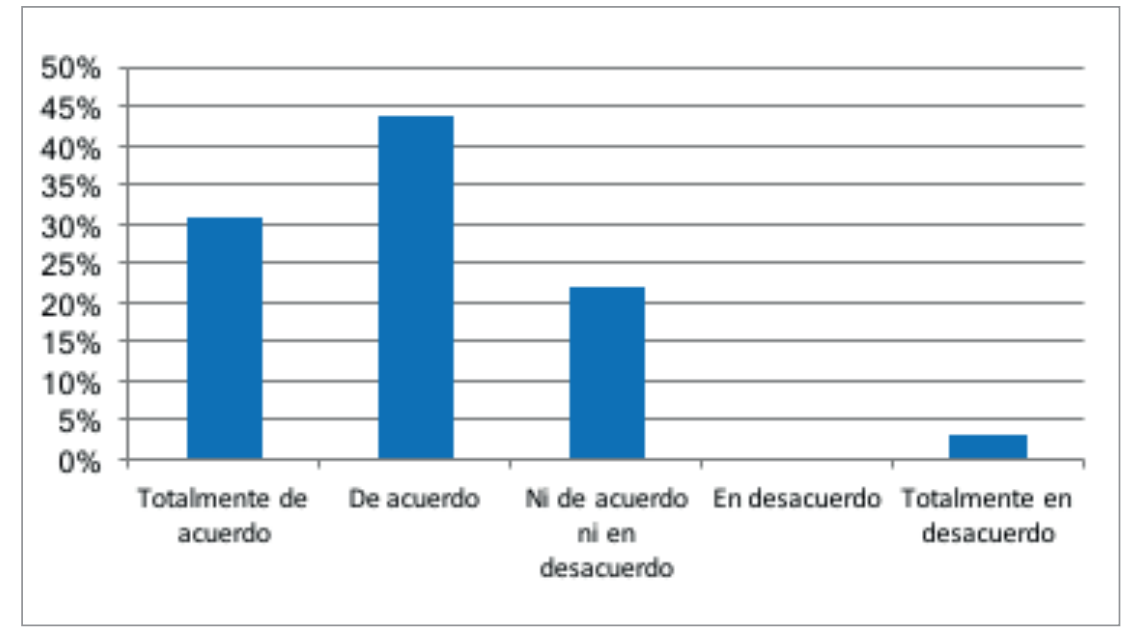

Figura 5. El acceso abierto incrementa las consultas y descargas 
En lo que respecta a la valoración de la calidad de los contenidos (figura $6)$, hay que destacar que tan sólo un $6 \%$ de los editores considera que la publicación en acceso abierto está pensada para contenidos de menor calidad. Por el contrario, el grupo mayoritario (50\%) considera que no existe ninguna relación entre acceso abierto y poca calidad y un $44 \%$ de las editoriales afirman que no están ni de acuerdo ni en desacuerdo. En este caso no existe correlación entre publicar en abierto y atribuir menor calidad a los contenidos.

En los comentarios quedan bien claros los argumentos sobre esta cuestión: la calidad científica del contenido no depende del grado de accesibilidad sino de otros parámetros y, por otro lado, el proceso de edición seguido para la publicación es idéntico para los títulos en acceso abierto y para los que no lo son. Los comentarios negativos aluden a una supuesta falta de revisión de los títulos y al tipo de obra que se publica en acceso abierto.

En relación con la pregunta sobre si los libros publicados en acceso abierto perjudican la venta de las mismas monografías en otros formatos encontramos que el $45 \%$ de las editoriales opinan que es difícil que un usuario que pueda obtener un libro de manera gratuita opte por pagar por él. Tan sólo el $10 \%$ de los editores estaría en desacuerdo con la afirmación siendo la UPV uno de ellos ya que afirma que han publicado libros que aun teniendo la versión electrónica se siguen vendiendo más en papel por su tema y contenido. Los comentarios expresan, en general, las dudas y la ausencia de datos que existen en esta cuestión. El perjuicio de las ventas se argumenta en base a que no se compra aquello que se puede obtener gratis, aunque se introduce el matiz de que la lectura online es menos perjudicial (incluso puede llegar a ser positiva para las ventas) que cuando existen posibilidades de descargar la obra. Otras

consideraciones que se introducen en la discusión se refieren al hecho de no poder generalizar sobre esta cuestión ya que en algún tipo de obra (manuales) no se perjudicaría la venta.

Respecto al futuro de la publicación en acceso abierto, algunos entrevistados (URV, UPC) valoran positivamente las perspectivas para las monografías dado que consideran que podrían seguir el mismo camino que las revistas. Destacan que los nuevos usuarios de monografías tienen unas costumbres diferentes al profesorado tradicional, y eso hace que cada vez más se publique en formato digital, según indica la UPC:

"Yo creo que sí porque al final las cosas caen por su propio peso. Si las revistas funcionan, las monografías también pueden funcionar. Llegaremos porque es lo más práctico, porque es hacia donde vamos, sobre todo para las nuevas generaciones, que trabajan de manera diferente a los catedráticos que hay ahora, que quieren el formato papel".

Otras, como la UPV, afirman que el proceso será más lento, tal vez debido a la extensión que ocupa una monografía, en comparación con un artículo, o porque el formato pdf, pensado para imprimir, no es muy ergonómico en el medio digital. El editor de la UCM cree que el paso de las monografías al acceso abierto será más lento que el de las revistas debido a cuestiones económicas y también a la reticencia que percibe en algunos autores a que sus trabajos sean gratuitos desde el primer momento.

Por otro lado, existe un consenso general en lo que respecta a los hábitos de los nuevos usuarios de monografías. Éstos tienen usos y costumbres diferentes al profesorado tradicional, y eso hace que cada vez más se publique en formato digital, como decían desde la UPC. 


\subsection{Financiación}

La viabilidad económica de los proyectos editoriales constituye lógicamente un aspecto fundamental y del máximo interés para los editores. El modelo de acceso abierto debe definir con claridad las vías posibles de financiación. Antes ya hemos preguntado sobre el posible impacto del acceso abierto en el descenso de ventas y hemos constatado sus dudas.

Poniendo el foco en los sistemas de financiación de monografías en acceso abierto, se ha querido recoger la opinión de las editoriales sobre los modelos predominantes:

- financiación institucional;

- por parte del autor;

- por parte de la editorial; y

- micromecenazgo.

Se ha preguntado si consideran estos modelos muy efectivos, bastante efectivos, efectivos, poco efectivos o nada efectivos. Para resumir la opinión de las editoriales sobre estos modelos y facilitar su interpretación, hemos reducido a dos los cinco parámetros de valoración: Efectivo (muy efectivo, bastante efectivo, efectivo) y No efectivo (poco efectivo y nada efectivo).

La forma reconocida mayoritariamente como la más efectiva por parte de las editoriales es la financiación institucional, en donde consta un saldo favorable del $82 \%$ (91\% de editoriales opinan que es muy efectiva, bastante efectiva o efectiva y tan sólo $9 \%$ que es poco o nada efectiva). Le sigue la financiación a cargo del presupuesto de la editorial, con un saldo del $32 \%$ y la financiación por parte del autor, con un saldo del $20 \%$. En el otro extremo - la poca efectividadencontramos el micromecenazgo, con un saldo negativo del $12 \%$.

Está claro que la financiación institucional es la vía más efectiva. Esto también se traslucía en las entrevistas. Para conseguir que esta opción sea realmente viable los editores destacan que la universidad debería invertir en la publica-

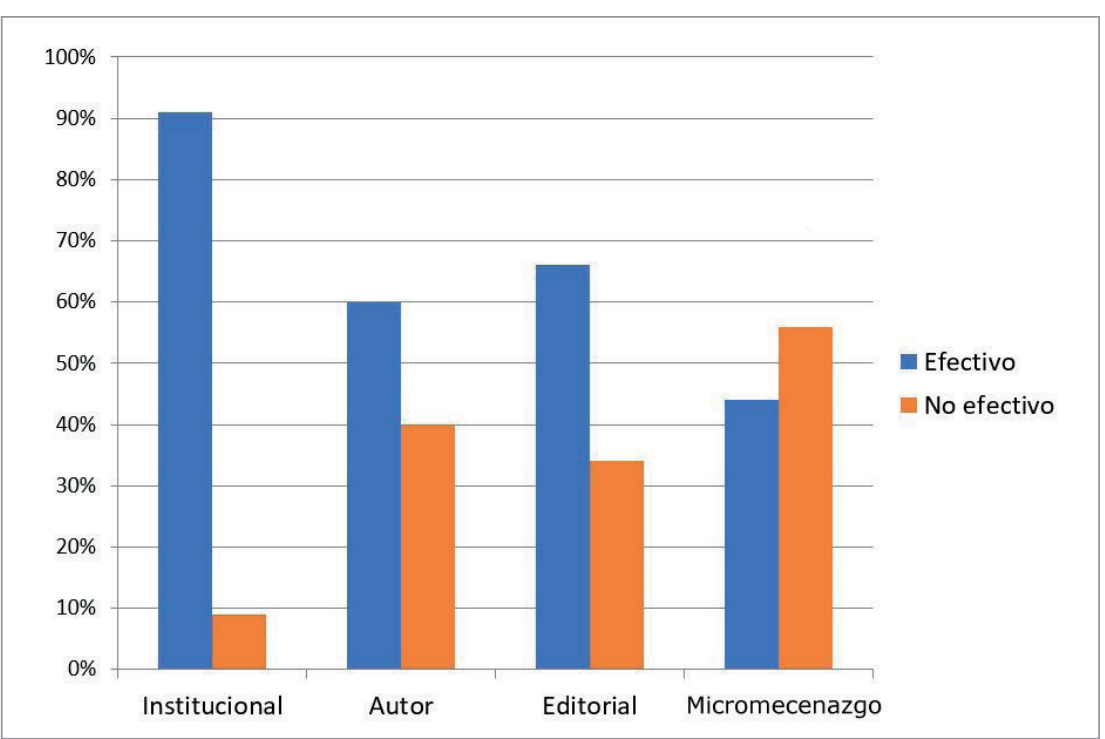

Figura 8. Valoración de los modelos de financiación ción y no tan sólo en la compra de libros (URV) y que la editorial tiene que ser considerada, desde el rectorado, de una manera similar a la biblioteca, es decir, un servicio que pone a disposición unos recursos ofrecidos gratuitamente (UPV).

\section{La financiación institucional es la vía más efectiva}

Respecto al pago por parte de los autores, algunos editores recuerdan que se tiene que sufragar mediante las partidas provenientes de los proyectos de investigación, ya que la publicación es la parte final del proceso de investigación. Esta vía de financiación es también aceptada por parte de editoriales poco proclives al acceso abierto, tal y como confirma la editora de la UOC cuando indica que:

"si un autor viene con el dinero para pagar los costes de edición, lo publicamos en abierto"

lo cual les sirve para ofrecer obras que tienen calidad pero que no forman parte de su línea editorial. En este contexto, todos los editores entrevistados defienden los criterios de calidad cuando insisten en que:

"aunque el autor venga con el dinero, si el libro no es bueno no se publica".

Además de conocer la opinión sobre las vías de financiación más utilizadas, también se ha preguntado por otras vías de financiación conocidas, citándose la coedición o el patrocinio, aunque con un peso residual. El representante de la URV hace notar que también se podría contar con la aportación económica de la biblioteca dado que las publicaciones en acceso abierto suponen un ahorro en su partida de adquisiciones.

Las editoriales que publican en acceso abierto tienen claro que las universidades públicas deben ofrecer en abierto todas sus publicaciones científicas para que puedan ser consultadas y reutilizadas por los ciudadanos. Sostienen que hacen una labor pública de transmisión del conocimiento y que invierten en cultura. Hasta ahora estos servicios editoriales son sostenibles, ahora bien, tal y como comenta la UPC, será necesario esperar para ver cómo evoluciona y confirmar si serán o no sostenibles para siempre. Estos buenos niveles de autofinanciación se han conseguido definiendo pocas colecciones, realizando tiradas ajustadas, estableciendo instrucciones de autoedición para los autores, vendiendo versiones impresas, etc. Estas acciones se ven facilitadas porque buena parte de su catálogo son manuales para estudiantes. Según algunos editores, como la UV, cuando se publica para audiencias más amplias es difícil aplicar estos criterios.

Las editoriales que no publican en acceso abierto comentan que deben 
tener ingresos para poder seguir publicando y compitiendo con el resto de las editoriales. Es el caso de la UOC que pertenece a una fundación privada y, por tanto, tiene una visión más empresarial del modelo de negocio.

\subsection{Políticas}

En estos momentos el acceso abierto está asumido por las agencias de financiación de la investigación y también por muchas universidades que han aprobado directrices o normativas para promover este modelo entre su comunidad. En un texto anterior nos referimos a la existencia de políticas de apoyo en las universidades españolas (Abadal et al., 2013) y en el directorio Melibea se pueden encontrar 37 políticas sobre acceso abierto aprobadas por universidades españolas.

http://www.accesoabierto.net/politicas

A la pregunta sobre la existencia en la universidad de alguna política o reglamentación respecto a la publicación de libros en acceso abierto, un $75,5 \%$ de las editoriales han respondido que sí y un $26,5 \%$ que no. Se pone de manifiesto que la mayor parte de las universidades han establecido una política respecto de esta cuestión. De todas formas, y como apunta el representante de la URV, en muchas universidades los mandatos o políticas sobre acceso abierto no han sido comentados con el servicio de publicaciones ni han generado, por tanto, un plan de actuación conjunto de los diversos servicios implicados.

En los comentarios de las editoriales quedan claras las diferencias de contenido de estas políticas. En algún caso se indica que se pueden publicar libros en acceso abierto cuando ha vencido su tiempo de comercialización, que se puede publicar inmediatamente en acceso abierto si el autor quiere, o que se debe publicar en el repositorio de la universidad un copia electrónica de cualquier publicación académica (artículo, comunicación, libros, etc.) en un plazo no superior a los seis meses de publicación, siempre teniendo en cuenta las condiciones establecidas por la editorial que publica. En otros casos se comenta que la política existente no se refiere a todos los documentos en general, sino tan sólo a las tesis.

La UPV reivindica el papel activo de las editoriales en el establecimiento de políticas de acceso abierto:

"Los servicios editoriales de las universidades debemos hacer entender al rectorado que somos un servicio público, mantenido por fondos públicos, y que nuestra labor es ayudar a transformar la información en conocimiento, saber comunicar y difundir, ayudar a los creadores de contenidos y poner en el mercado libre el conocimiento para que pueda ser reutilizado".

\section{Discusión y conclusiones}

Hay que tener presente que las editoriales universitarias son las más proclives o, al menos, las que tienen unas condiciones objetivas mejores para facilitar la difusión en acceso abierto de sus publicaciones. Esto puede conducirlas a una transición total o parcial hacia este modelo de difusión. Por este motivo se ha llevado a cabo el estudio en este tipo de editor.

Se constata que el concepto de acceso abierto en monografías es bastante amplio. En este sentido, a tenor de las respuestas, los editores incluyen bajo este término a los títulos accesibles después de un embargo temporal, a los gratuitos y a los que incluyen también una licencia Creative Commons. Desde nuestro punto de vista, en esta fase inicial de desarrollo del modelo es importante la flexibilidad y considerar todos los niveles de apertura, un poco al estilo del documento How open is it? (Sparc; Plos; Caspa, 2014).

El número de editoriales que en 2017 publican monografías en acceso abierto es destacable (59\%) y los datos son mejores aún si se considera el porcentaje (28\%) que lo estaba valorando en 2016.

En lo que respecta a la oferta actual, aunque el número de editoriales que publican en acceso abierto es notable, el número de títulos no es relevante y, lo que es más importante, no se dispone de plataformas que permitan un acceso integrado y una buena visibilidad de la oferta existente como es el caso de Oapen o DOAB en el ámbito internacional. Según los datos de la encuesta UNE (2016), la mayor parte de los títulos en acceso abierto (62,2\%) se comparte en la web de las editoriales y también en el repositorio general de la universidad. En algún caso, como Libros UM, se ha creado un portal específico. De todas formas, no existe ningún portal global similar a los citados a nivel internacional que agrupe todos los títulos en abierto. Quizá la propia UNE podría asumir un papel de coordinación relevante en este sentido.

\section{Aunque el número de editoriales que publican en acceso abierto es notable, el número de títulos no lo es, y no se dis- pone de plataformas que permitan un acceso integrado y una buena visibilidad de la oferta}

El camino a recorrer por parte de las monografías está surtido de mayor número de dificultades que el seguido por las revistas:

- el coste de publicación, mucho más alto que para las revistas;

- un cierto apego aún al papel en diversas áreas, particularmente las humanidades o el derecho.

En lo que respecta a las valoraciones sobre el acceso abierto se destaca positivamente el hecho de incrementar las consultas, y no se considera que tenga relación con la calidad de los contenidos. En estas dos cuestiones se detecta una correlación entre opiniones positivas y haber publicado siguiendo este modelo. Así pues, las editoriales que publican en acceso abierto tienen una visión positiva de sus ventajas mientras que las que no lo hacen no ven argumentos a favor de este modelo de difusión. Existe más disparidad de valoración sobre la compatibilidad del acceso abierto con la explotación comercial impresa, lo cual es lógico.

La financiación constituye, sin ninguna duda, el elemento fundamental que dificulta en estos momentos el crecimiento de este modelo de publicación y, a la vez, la principal preocupación de los editores. Elliott también insiste en esta cuestión cuando señala como una de las conclusiones de su estudio: 
"Apoyamos un modelo de financiación universitaria para la publicación de monografías digitales [...] Apoyamos la publicación en acceso abierto de formatos académicos extensos" (Elliott, 2015).

Los editores españoles destacan de manera relevante como mejor opción la financiación institucional por parte de la propia universidad. Los entrevistados insisten en esta cuestión e indican que la biblioteca y la universidad deberían invertir en la publicación o que la editorial tiene que ser considerada, desde el rectorado, como la biblioteca, que ofrece unos servicios que no se cobran.

También se tiene en cuenta la vía de financiación por parte del autor cuando se invoca que la publicación tiene que ser vista como parte de la investigación y se tiene que pagar con cargo a los proyectos de investigación. Las agencias de financiación de proyectos, así como H2020, contemplan claramente la imputación de costes para la publicación de monografías y esta oportunidad no puede pasar desapercibida por los editores universitarios. Se trata de una vía muy minoritaria aún en España con una diferencia clara con la propuesta de Greco y Wharton (2008) para Norteamérica, basada fundamentalmente en las aportaciones de los autores. En esta línea, uno de los ejemplos pioneros y más conocidos es el programa Luminos, de la University of California Press, en el cual se solicitan 7.500\$ al autor para sufragar los costes editoriales.

La mayoría de las universidades dispone de alguna recomendación o mandato respecto del acceso abierto a la ciencia. Por tanto, es necesario que las editoriales se alineen y vayan acordes con la misión de la universidad. En ello también coincidía Shearer (2010) en su estudio:

"Sin un fuerte apoyo institucional, ha sido difícil para las editoriales adaptarse a unas circunstancias tan cambiantes".

Sin embargo no es tan sencillo llevar a cabo estas actuaciones y se necesita el apoyo directo de los rectores para que se confirme el apoyo económico de la universidad, sin el cual no se puede llevar a cabo una política de acceso abierto. No se analizan las implicaciones que las políticas de la universidad tienen para el servicio de publicaciones. Así pues, queda clara y patente la contradicción existente entre las declaraciones teóricas y el apoyo financiero.

Nos encontramos en una fase de transición en la cual los editores deben planificar bien sus próximos pasos. También es evidente que la principal preocupación de los editores en estos momentos reside en la obtención de recursos para evolucionar de un modelo basado en el pago por parte de los lectores a otra que debe descansar en la financiación de los autores o de las propias universidades. No va a ser fácil convencer a ninguno de ellos, pero el contexto y la presión del entorno juegan en favor del acceso abierto y ésta va a ser la principal baza que tienen a su favor los editores.

Uno de los retos actuales, que no ha sido tratado en nuestro estudio pero que ha sido especialmente comentado tanto en la encuesta como en las entrevistas, es establecer un sistema de evaluación de la calidad, un tema en el cual destacan los estudios de Giménez-Toledo (2016) y Giménez-To- ledo y Tejada-Artigas (2012, 2014). Las presiones derivadas de la evaluación del profesorado son las que condicionan la producción editorial y las preocupaciones actuales de los editores (establecer un sello de calidad UNE, los criterios de la Aneca y el proceso de evaluación del profesorado, etc.).

\section{Notas}

1. Consejo Superior de Investigaciones Científicas (CSIC), Universidad Complutense de Madrid (UCM), Universitat Oberta de Catalunya (UOC), Universitat Politècnica de Catalunya (UPC), Universitat Politècnica de València (UPV), Universitat Rovira i Virgili (URV), Universitat de València (UV).

\section{Agradecimientos}

A Lluís Pastor presidente de la UNE (2012-2016) y Alicia Buil, secretaria de la misma entidad.

A los editores universitarios encuestados y especialmente a Meritxell Anton (UB), José Luís Canet (UV), Roser Leal (UOC), Jaume Llambrich (URV), Antonio López-Fonseca (UCM), María-Remedios Pérez-García (UPV), Jordi Prats (UPC) y Ramón B. Rodríguez (CSIC).

Este estudio se ha realizado en el marco del proyecto Acceso abierto a la ciencia en España (CSO2014-52830-P) financiado por el Plan Estatal de I+D del Ministerio de Economía y Competitividad.

\section{Referencias}

Abadal, Ernest; Ollé, Candela (2012). La edición universitaria en el contexto de la ciencia abierta. Barcelona: Editorial UOC. ISBN: 9788490299999

Abadal, Ernest; Ollé, Candela; Abad-García, Francisca; Melero, Remedios (2013). "Políticas de acceso abierto a la ciencia en las universidades españolas". Revista española de documentación científica, v. 36, n. 2, e007. https://doi.org/10.3989/redc.2013.2.933

Archambault, Eric; Didier, Amyot; Deschamps, Philippe; Nicol, Aurore; Rebout, Lise; Roberge, Guillaume (2013). Proportion of open access peer-reviewed papers at the European and world levels: 2004-2011. Montréal: Science-Metrix. https://goo.gl/FQalXH

Björk, Bo-Christer; Welling, Patrik; Laakso, Mikael; Maijlender, Peter; Hedlund, Turid; Guönason, Guöni (2010). "Open access to the scientific journal literature: situation 2009". PLOS ONE, v. 5, n. 6.

https://doi.org/10.1371/journal.pone.0011273

Chen, Xiaotian (2014). "Open access in 2013: reaching the $50 \%$ milestone". Serials review, v. 40, n. 1.

https://goo.gl/KSpo75

https://doi.org/10.1080/00987913.2014.895556

Collins, Ellen; Milloy, Caren (2016). Oapen-UK final report: A five-year study into open access monograph publishing in the humanities and social sciences. JISC. https://goo.gl/B1OiCZ

Cordón-García, José-Antonio (2014). “Nuevas tendencias en el libro electrónico universitario". Anuario ThinkEPI, v. 8, pp. 269-276. 
https://recyt.fecyt.es/index.php/ThinkEPI/article/view/29592

Cordón-García, José-Antonio; Gómez-Díaz, Raquel; Alonso-Arévalo, Julio; Alonso-Berrocal, José-Luis (2013). El ecosistema del libro electrónico universitario. Salamanca: Universidad de Salamanca, UNE. ISBN: 9788490122853

Deegan, Marilyn (2017). Academic book of the future project report: A report to The AHRC \& The British Library. London: The British Library.

https://academicbookfuture.files.wordpress.com/2017/06/ project-report_academic-book-of-the-future_deegan3.pdf

Elliott, Michael (2015). "The future of the monograph in the digital era: A report to the Andrew W. Mellon Foundation". The journal of electronic publishing, v. 18, n. 4.

https://doi.org/10.3998/3336451.0018.407

Ferwerda, Eelco (2014). “Open access monograph business models". Insights, v. 27, pp. 35-38.

https://doi.org/10.1629/2048-7754.46

Ferwerda, Eelco; Pinter, Frances; Stern, Niels (2017). A landscape study on open access and monographs: Policies, funding and publishing in eight European countries. Norway: Knowledge Exchange.

https://doi.org/10.5281/zenodo.815932

Ferwerda, Eelco; Snijder Ronald; Adema, Janneke (2013). Oapen-NL: a project exploring open access monograph publishing in the Netherlands. Final report. The Hague: Oapen; SURF; NWO.

http://oapen.org/download?type=export\&export=oapennl-final-report

Giménez-Toledo, Elea (2016). "Assessment of journal \& book publishers in the humanities and social sciences in Spain". In: Oschner, Michael; Hug, Sven; Dieter-Daniel, Hans. Research assessment in the humanities. Springer International Publishing, pp. 91-102. ISBN: 9783319290164 http://link.springer.com/chapter/10.1007/978-3-319-29016-4_8/ fulltext.html

Giménez-Toledo, Elea; Tejada-Artigas, Carlos-Miguel (2012). "Valoración de editoriales especializadas en comunicación, biblioteconomía y documentación: encuesta a profesores e investigadores". El profesional de la información, v. 21, n. 1, pp. 50-62.

https://doi.org/10.3145/epi.2012.ene.07

Giménez-Toledo, Elea; Tejada-Artigas, Carlos-Miguel (2014).
"Proceso de publicación, calidad y prestigio de las editoriales científicas en educación”. Educación XXI, v. 18, n. 1.

https://doi.org/10.5944/educxx1.18.1.12310

Greco, Albert; Wharton, Robert-Michael (2008). "Should university presses adopt an open access [electronic publishing] business model for all of their scholarly books?". En: Chan, Leslie; Mornati, Susanna. Elpub2008. Open Scholarship: Authority, community, and sustainability in the age of web 2.0. Proceedings of the $12^{\text {th }}$ Int l conf on electronic publishing, Toronto, Canada, 25-27 June 2008, pp. 49-164. ISBN: 9780772763150

https://elpub.architexturez.net/system/files/pdf/149_ elpub2008.content.pdf

Jubb, Michael (2017). Academic books and their future: $A$ report to The AHRC and The British Library. London: The British Library.

https://academicbookfuture.files.wordpress.com/2017/06/ academic-books-and-their-futures jubb1.pdf

Montgomery, Lucy (2015). “Knowledge unlatched: A global library consortium model for funding open access scholarly books. Full report on the proof of concept pilot 2014". Journal of cultural science, v. 7, n. 2.

https://goo.gl/cJ43Kh

Polo-Pujadas, Magda (coord.) (2007). Innovación y retos de la edición universitaria. Madrid: UNE. ISBN: 9788496487277 https://goo.gl/xiwqF9

Shearer, Kathleen (2010). A review of emerging models in Canadian academic publishing. University of British Columbia Library.

https://goo.gl/KnuEzF

Sparc; Plos; Caspa (2014). How open is it? Espectro de acceso abierto. Publishing and Academic Resources Coalition \& Public Library of Science.

https://www.plos.org/files/HowOpenls/t_Spanish.pdf

Taylor, Donald; Morrison, Heather; Owen, Brian; Vézina, Kumiko; Waller, Andrew (2013). “Open access publishing in Canada: Current and future library and university press supports". Publications 2013, v. 1, pp. 27-48.

https://doi.org/10.3390/publications1010027

UNE (2016). Las editoriales universitarias en cifras 2016. Madrid: UNE.

https://goo.gl/lbCdMw 


\section{Anexo: Encuesta a editores sobre el acceso abierto en monografías \\ Datos descriptivos}

1. Nombre de la editorial

2. Nombre del editor

3. ¿Cuantos libros publicó en 2015?

Menos de 50

50-100

Más de 100

4. ¿Qué porcentaje aproximado fueron en formato papel, digital o en ambos?

\begin{tabular}{|l|l|l|l|l|}
\cline { 2 - 5 } \multicolumn{1}{l|}{} & $0-25$ & $26-50$ & $51-75$ & $75-100$ \\
\hline Papel & & & & \\
\hline Digital & & & & \\
\hline Ambos & & & & \\
\hline
\end{tabular}

5. ¿Cuantos libros prevé publicar en 2016?

Menos de 50

50-100

Más de 100

6. ¿Qué porcentaje aproximado fueron en formato papel, digital o en ambos?

\begin{tabular}{|l|l|l|l|l|}
\hline \multicolumn{1}{l|}{$0-25$} & $26-50$ & $51-75$ & $75-100$ \\
\hline Papel & & & & \\
\hline Digital & & & & \\
\hline Ambos & & & & \\
\hline
\end{tabular}

Valoración del acceso abierto en a publicación de monografías

7. Publicar libros en acceso abierto incrementa las consultas y descargas Totalmente en desacuerdo

En desacuerdo

Ni de acuerdo ni en desacuerdo

De acuerdo

Totalmente de acuerdo

8. ¿Por qué?

9. Los libros que se publican en acceso abierto tienen menor calidad Totalmente en desacuerdo

En desacuerdo

Ni de acuerdo ni en desacuerdo

De acuerdo

Totalmente de acuerdo

10. ¿Por qué?

11. Los libros publicados en acceso abierto perjudican la venta de la misma monografía en otros formatos

Totalmente en desacuerdo

En desacuerdo

$\mathrm{Ni}$ de acuerdo ni en desacuerdo

De acuerdo

12. ¿Por qué?

\section{Publicaciones en acceso abierto}

13. ¿Publicó libros en acceso abierto en 2015 ?

$\mathrm{Si}$

No

Si ha contestado NO, puede pasar a la pregunta núm. 16

14. ¿Cuantos libros en acceso abierto publicó en 2015 ?

15. ¿'De qué disciplinas eran?

Artes plásticas y escénicas

Humanidades

Ciencias sociales

Ciencias experimentales

Matemáticas y ciencias físicas

Ciencias de la vida

Ciencias de la salud

Ingeniería
16. ¿Ha publicado libros en acceso abierto en 2016 ? $\mathrm{Si}$

No

Si ha contestado NO, puede pasar a la pregunta núm. 19

17. ¿Cuantos libros en acceso abierto prevé publicar en 2016?

18. ¿De qué disciplinas?

Artes plásticas y escénicas

Humanidades

Ciencias sociales

Ciencias experimentales

Matemáticas y ciencias físicas

Ciencias de la vida

Ciencias de la salud

Ingeniería

19. ¿Tiene previsto incluir la publicación de libros en acceso abierto en 2017 ? $\mathrm{Si}$

No

Lo estamos valorando

20. ¿Pone en acceso abierto los libros publicados, una vez transcurrido el período de explotación comercial indicado en el contrato de edición?

Si

No

Lo estamos valorando

21. ¿Por qué?

\section{Modelos y políticas de publicación en acceso abierto}

22. ¿Qué modelos de financiación cree que son más efectivos para publicar en acceso abierto?

\begin{tabular}{|l|l|l|l|l|}
\hline \multicolumn{1}{|c|}{$\begin{array}{c}\text { Nada } \\
\text { efectivo }\end{array}$} & $\begin{array}{c}\text { Poco } \\
\text { efectivo }\end{array}$ & Efectivo & $\begin{array}{c}\text { Bastante } \\
\text { efectivo }\end{array}$ & $\begin{array}{c}\text { Muy } \\
\text { efectivo }\end{array}$ \\
\hline $\begin{array}{l}\text { Financiación institu- } \\
\text { cional (universidad, } \\
\text { etc.) }\end{array}$ & & & & \\
\hline $\begin{array}{l}\text { Financiación por } \\
\text { parte del autor }\end{array}$ & & & \\
\hline $\begin{array}{l}\text { Financiación por } \\
\text { parte del presupuesto } \\
\text { de la editorial }\end{array}$ & & & & \\
\hline Micromecenazgo & & & & \\
\hline
\end{tabular}

23. ¿Si conoce alguna otra vía de financiación, puede describirla?

24. ¿Existe en su universidad alguna política o reglamentación respecto a la publicación de libros en acceso abierto

$\mathrm{Si}$

No

Si ha contestado NO, puede pasar a la pregunta núm. 26

25. ¿Puede hacer una breve descripción de esta política o reglamentación?

26. ¿Puede indicar una experiencia positiva de publicación de una obra o colección determinada en acceso abierto?

27. ¿Puede indicar una experiencia ngativa de publicación de una obra o colección determinada en acceso abierto?

\section{Comentario final}

28. ¿Quiere hacer algun comentario o recomendación final?

29. ¿Estaría dispuesto a participar en una entrevista más detallada sobre estas cuestiones?

$\mathrm{Si}$

No

30. Puede adjuntar un correo electrónico para contactar con usted para una posible entrevista.

MUCHAS GRACIAS POR SU COLABORACIÓN 